\title{
富硒螺旋藻中含硒藻蓝蛋白的纯化、结晶 及初步晶体学研究*
}

\section{李乐农 ${ }^{(1)} \quad$ 张季平 $^{(2)}$ 江 涛 $^{(2)}$ 郭宝江 ${ }^{(1)}$ 常文瑞 (2) 梁栋材 ${ }^{(2) * *}$}

(1)华南师范大学生物技术研究所, 广州 510631; (2)中国科学院生物物理研究所生物大分子国家重点实验室, 北京 100101)

\begin{abstract}
摘要从富硒螺旋藻中提取含硒藻蓝蛋白, 经凝胶色谱和离子交换色谱纯化, 应用 悬滴气相扩散法, 采用 $\left(\mathrm{NH}_{4}\right)_{2} \mathrm{SO}_{4}$ 和 PEG4000 作沉淀剂, 获得了该蛋白质晶体的两种晶 型. 晶型 I 属于单斜晶系, 晶胞参数 $a=10.80 \mathrm{~nm}, b=11.70 \mathrm{~nm}, c=18.40 \mathrm{~nm}, \beta=90.2^{\circ}$, 晶 体空间群属于 $\mathrm{P} 22_{1}$. 单位晶胞中每个晶体学不对称单位含 12 个 $(\alpha \beta)$ 单体, 晶体衍射的 最高分辨率达 $0.28 \mathrm{~nm}$. 晶型 II 为六方晶系, 晶胞参数为 $a=b=15.5 \mathrm{~nm}, c=4.03 \mathrm{~nm}$, 晶体空间群属于 $\mathrm{P}_{3}$. 晶体衍射的最高分辨率达 $0.28 \mathrm{~nm}$. 单位晶胞中每个晶体学不对 称单位含 1 个 $(\alpha \beta)$ 单体. 对分子在晶体中的可能堆积方式进行了讨论.
\end{abstract}

关键词藻蓝蛋白 晶体生长 晶体学研究 $X$ 射线衍射

硒是生命的必需微量元素 ${ }^{[1]} .1973$ 年, Rotruck 等人 ${ }^{[2]}$ 发现哺乳动物中的第 1 个含硒酶即谷 胱甘肽过氧化物酶. 此后, 有关硒在生物体内的代谢及功能的研究越来越受到关注. 目前对硒 的研究已深入到人和动物的营养、生理作用, 以及抗癌机制研究. 在动物和微生物体内亦已 相继发现许多含硒酶和硒蛋白, 有些已深入到分子水平阐明其作用机理. 研究表明 ${ }^{[3,4]}$, 硒蛋 白合成过程中, UGA 是蛋白质硒半胱氨酸的密码子, 硒蛋白以硒半胱氨酸形式掺入到蛋白质 中. 有关植物硒蛋白的研究较少, 但由于有机硒的重要医疗保健作用, 目前人们试图通过生物 富集的方法, 将无机硒转化为生物活性高、易为人和动物吸收利用的有机硒. 据报道 ${ }^{[5,6]}$, 植 物对硒酸盐的吸收与硫酸盐相似, 硒酸盐经吸收后能形成有机硒化物, 而硒化物主要是以各 种含 R-Se-R' 形式的氨基酸. 国内外许多研究者采用根部施硒肥或者叶面喷硒，可提高植物 体含硒量, 并认为硒对植物抗氧化、蛋白质合成代谢等许多生理功能有影响. 孙景信等人 ${ }^{[7]}$ 报 道, 采用含硒介质培养冬小麦幼苗, 发现外源硒存在于过氧化物同工酶中. 对藻类和硒酵母的 研究表明 ${ }^{[8-10]}$, 硒主要分布在蛋白质中. 但是, 迄今为止, 有关植物体内含硒蛋白质大分子的 存在多限于实验性的推断，还没有更直接的证据表明外源硒进入蛋白质的存在状况，因而不 能阐明硒掺入植物体蛋白质中的机理及对植物体生理功能的影响.

藻蓝蛋白是红藻和蓝绿藻的一类重要捕光色素蛋白复合物, 在光合作用过程中对光能的 吸收、传递起重要作用, 对其三维结构的研究有助于阐明光合作用机理. 此外, 藻蓝蛋白还可

1999-08-27 收稿, 2000-02-28 收修改稿

* 国家自然科学基金重点资助项目(批准号: 39270156)和广东省及广州市重点资助科研攻关课题

***联系人(E-mail: dcliang@sun5.ibp.ac.cn) 
作为细胞和分子的苂光标记物, 在免疫检测、苂光显微术、流式细胞仪技术等方面有很好的 应用前景 ${ }^{[11]}$. 此方面已有许多文献报道. 在蓝绿藻、螺旋藻方面, Moreno 和 Brejc 等人 ${ }^{[11,12]}$ 对 藻胆蛋白做了很多研究. 迄今为止, 在所有藻类已测定结构的藻胆蛋白中: 藻红蛋白有来自 Porphyridium sordidum 的 B-PE ${ }^{[13]}$, Polysiphonia urceolata 的 R-PE ${ }^{[14,15]}$, Porphyridium cruentum 的 b-PE ${ }^{[16]}$ 和 B-PE ${ }^{[17]}$; 藻红蓝蛋白有来自 Mastigocladus laminosus 的 $\mathrm{PEC}^{[18]}$; 藻蓝蛋白有来自 Mastigocladus laminosus 的 C-PC ${ }^{[19]}$, Agmenellum quadruplicatum 的 C-PC ${ }^{[19]}$, Fremyella diplosiphon 的 C-PC ${ }^{[20]}$, Anabaena variabilis 的 C-PC ${ }^{[17]}$; 别藻蓝蛋白有来自 Porphyra yezoensis 的 $\mathrm{APC}^{[21]}$ 和 Spirulina platensis 的 $\mathrm{APC}^{[12]}$, 但是, 未见有螺旋藻藻蓝蛋白三维结构测定的报道.

螺旋藻是一种有丰富营养价值的保健食品资源, 但其硒含量极微, 利用生物富集转化方 法可提高藻体含硒量及增强其生理保健功能. 而藻蓝蛋白作为藻体内的主要营养成分, 经研 究发现, 富硒螺旋藻中的藻蓝蛋白有硒存在. 目前还不清楚硒是否经蛋白质合成过程掺入到 蛋白质中, 更不清楚它对螺旋藻的生理功能有何影响. 因此, 对富硒螺旋藻中藻蓝蛋白三维结 构的测定, 有助于研究硒在螺旋藻的藻蓝蛋白中的存在形式及其从分子水平对外源硒进入螺 旋藻㩰蓝蛋白的机理进行探讨, 并对阐明植物的硒营养代谢以及光合作用机理有着重要的理 论与实践意义.

本文报道了含硒藻蓝蛋白的制备、结晶及初步晶体学研究结果.

\section{1 实验与结果}

\section{1 富硒螺旋藻的培养}

钝螺旋藻(Spirulina platensis, 由深圳蓝藻公司提供)培养采用 Zarrouk 培养基, pH 8.5 9.5. 在 $1000 \mathrm{~mL}$ 三角瓶中加入 $800 \mathrm{~mL}$ 培养液, 将旺盛生长的藻种接种于有培养液的三角瓶中, 每 瓶接种量相同. 在培养到第 3 天后, 分别加入浓度为 $8 \%$ 的亚硒酸钠溶液, 使得三角瓶中的亚 硒酸钠浓度为 $600 \mathrm{mg} / \mathrm{L}$. 培养条件: 光照强度为 $50 \times 10^{-6} \mathrm{~lx} /\left(\mathrm{m}^{2} \cdot \mathrm{s}\right)$, 光照时间为 $12 \mathrm{~h}$, 温度 为 $28 \sim 35^{\circ} \mathrm{C}$, 由空气压缩泉供给氧气. 培养 $12 \mathrm{~d}$ 后, 收获藻体, 充分透析后将其冷冻干燥保存.

\section{2 藻蓝蛋白提取、纯化}

称 $10 \mathrm{~g}$ 干藻粉, 加 $200 \mathrm{~mL}$ 磷酸缓冲液 $(\mathrm{pH} 7.0)$ 浸泡, 置低温冰箱 $\left(-20^{\circ} \mathrm{C}\right)$ 冻融数次, 直至 全部细胞破碎. 随后离心 $20 \mathrm{~min}\left(3000 \mathrm{r} / \mathrm{min}, 4^{\circ} \mathrm{C}\right)$, 弃沉淀的藻泥和沉淀, 上清液用 $20 \%(w / v)$ 饱和硫酸铵沉淀; 离心 $20 \mathrm{~min}\left(3000 \mathrm{r} / \mathrm{min}, 4^{\circ} \mathrm{C}\right)$, 弃沉淀, 上清液用 $45 \%(w / v)$ 饱和硫酸铵沉淀; 离心 $20 \min \left(3000 \mathrm{r} / \mathrm{min}, 4^{\circ} \mathrm{C}\right)$, 弃上清液, 收集沉淀, 并溶于少量磷酸缓冲液( $\mathrm{pH}$ 7.0)中, $4^{\circ} \mathrm{C}$ 下 透析至平衡. 然后将蛋白质溶液离心 $10 \mathrm{~min}\left(13000 \mathrm{r} / \mathrm{min}, 4^{\circ} \mathrm{C}\right)$, 除去变性蛋白后, 上清液经 $\mathrm{Na}_{2} \mathrm{HPO}_{4}-\mathrm{NaH}_{2} \mathrm{PO}_{4}$ ( $\mathrm{pH}$ 7.0)缓冲液预平衡了的 DEAE 纤维素层析柱 $52(60 \times 2.5 \mathrm{~cm})$ 过柱, 采用 盐浓度梯度洗脱, 分别收集不同离子强度缓冲液的洗脱液, 立即进行光谱测定. 在室温 $\left(25^{\circ} \mathrm{C}\right)$ 用岛津 UV-3000 紫外分光光度计测定其吸收光谱(图 1), 根据 $A_{620} / A_{280}$ 收集蛋白质溶液. 在此 基础上再将所收集的蛋白质溶液过 Sephadex G-100 柱 $(60 \times 2.5 \mathrm{~cm})$, 并将 $A_{620} / A_{280}>3.5$ 的蛋 白质溶液管合并. 将上述已合并的蛋白质溶液再经 FPLC(MonoQ 柱)分离后, 进一步去掉杂蛋 白, 经 SDS 电泳, 测得藻蓝蛋白两个亚基的分子量为 $\alpha=19 \mathrm{ku}, \beta=21.5 \mathrm{ku}$ (图 2).

\subsection{Se 含量测定}

DAN-菼光法 ${ }^{[22]}$ : 取上述纯化的蛋白质样品 $1.2 \mathrm{~mL}$ (蛋白质浓度 $2.87 \mathrm{mg} / \mathrm{mL}$ )置于具塞三 


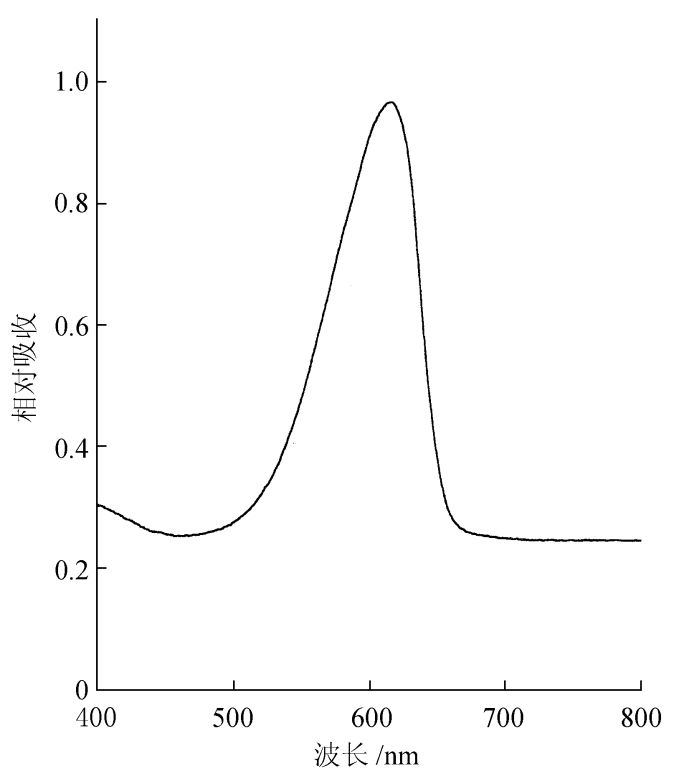

图 1 钝顶螺旋藻藻蓝蛋白的吸收光谱 $\left(25^{\circ} \mathrm{C}\right)$

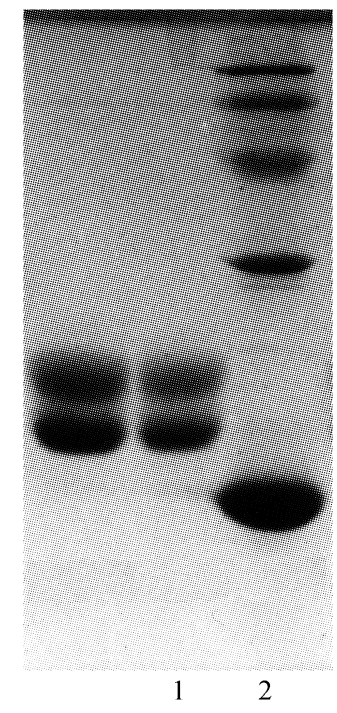

图 2 经 FPLC(MonoQ 柱)分离后钝顶螺旋藻藻蓝 蛋白的 SDS-PAGE

1 示藻蓝蛋白样品; 2 示标准蛋白 (从上到下): 磷酸化酶 B $(94 \mathrm{ku}) 、$ 牛血清白蛋白 $(67 \mathrm{ku})$ 、肌动蛋白 $(43 \mathrm{ku})$ 、碳酸酐 酶 $(30 \mathrm{ku}) 、 \alpha$-乳清蛋白(14.4 ku)

角瓶中, 经混合酸 $\left(\mathrm{HNO}_{3}: \mathrm{HClO}_{4}=4: 1\right)$ 消化后定容. 然后取一定量的消化液, 加适量盐酸着氨, EDTA, 甲酚红混合液后溶液呈深粉红色, 用氨溶液或浓盐酸调至略呈淡粉红橙色 $(\mathrm{pH}=1 \sim 2)$. 在暗室中, 定量加入新配制的 $0.1 \% 2,3$-二氨基萗, 在沸水浴上加热 $5 \mathrm{~min}$. 冷却后转入分液 漏斗中, 然后加入一定量的环己烷, 充分振摇, 静置分层, 将环己烷层倾入具塞小试管中, 用 RF-5400 苂光分光光度计测定其苂光强度, 同时做硒标准溶液(制作标准曲线)、空白和回收率 测定. 测定条件: 激发波长 $376 \mathrm{~nm}$, 发射波 长为 $520 \mathrm{~nm}$, 狭缝 $10 \mathrm{~nm}$, 测量池 $1 \mathrm{~cm}$. 在 此条件下测得样品与标准品的苂光强度, 并 计算出 $\mathrm{Se}$ 含量.

按上述方法测得藻蓝蛋白样品 $\mathrm{Se}$ 含量 为 $86.8 \mu \mathrm{g} / \mathrm{g}$ 蛋白.

\section{4 晶体生长及其 $\mathrm{X}$ 射线衍射研究}

\subsection{1 晶型 I 在室温避光的条件下采} 用气相悬滴扩散法, 按表 1 所列的晶体生长 条件, 经 4 5 周的时间获得了最大线度为 $0.65 \mathrm{~mm} \times 0.4 \mathrm{~mm} \times 0.15 \mathrm{~mm}$ 的晶体. 图 3 给出了晶体的外形照片, 将晶体装入石英薄 壁小管内, 使用 Mar Research Image Plate System 进行衍射数据的收集, 晶体衍射的最

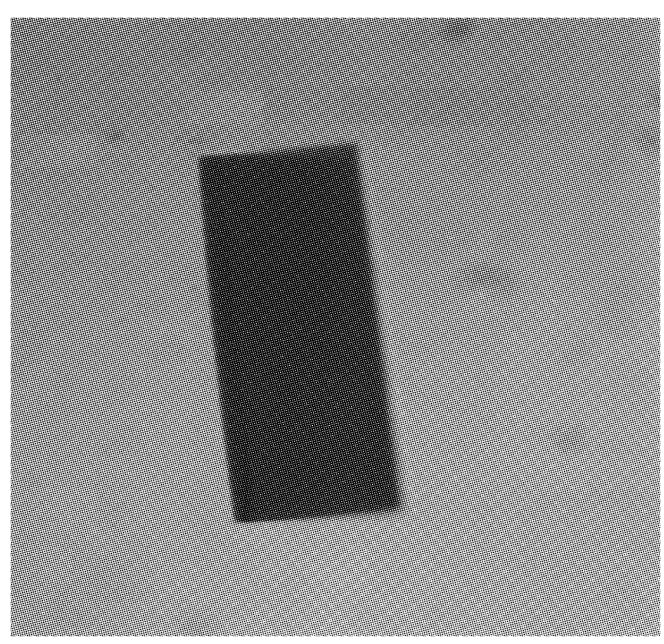

图 3 钝顶螺旋藻藻蓝蛋白晶型 I 的晶体外形照片 
表 1 钝顶螺旋藻藻蓝蛋白晶型 I 和 II 的晶体生长条件

\begin{tabular}{|c|c|c|}
\hline & 晶型 I & 晶型 II \\
\hline $\mathrm{A}$ 液 & $\begin{array}{l}\mathrm{MPD}^{\mathrm{a})} 2.5 \%(v / v) \\
\text { 饱和 }\left(\mathrm{NH}_{4}\right)_{2} \mathrm{SO}_{4} 16 \%(v / v) \\
\text { 饱和 } \mathrm{NaCl} 6 \%(v / v) \\
0.1 \mathrm{~mol} / \mathrm{L} \mathrm{Na} \mathrm{NPO}_{4}-\mathrm{NaH}_{2} \mathrm{PO}_{4} \\
\text { pH } 6.79\end{array}$ & $\begin{array}{l}\mathrm{PEG} 400013 \%(w / v) \\
\mathrm{MgCl}_{2} 0.4 \mathrm{~mol} / \mathrm{L} \\
\text { Hepes } 0.1 \mathrm{~mol} / \mathrm{L} \\
\mathrm{pH} 7.37\end{array}$ \\
\hline $\mathrm{B}$ 液 & $\begin{array}{l}11.0 \mathrm{mg} \text { 蛋白溶于 } 1 \mathrm{~mL} \\
0.1 \mathrm{~mol} / \mathrm{L} \mathrm{Na}_{2} \mathrm{HPO}_{4}-\mathrm{NaH}_{2} \mathrm{PO}_{4} \\
\text { pH } 6.79\end{array}$ & $\begin{array}{l}10.0 \mathrm{mg} \text { 蛋白溶于 } 1 \mathrm{~mL} \\
0.1 \mathrm{~mol} / \mathrm{L} \mathrm{Hepes} \\
\mathrm{pH} 7.37\end{array}$ \\
\hline 悬滴液 & $4 \mu \mathrm{L} \mathrm{A}$ 液 $+4 \mu \mathrm{L} \mathrm{B}$ 液 & $4 \mu \mathrm{L} \mathrm{A}$ 液 $+4 \mu \mathrm{L} \mathrm{B}$ 液 \\
\hline 扩散液 & $\begin{array}{l}\text { 饱和 }\left(\mathrm{NH}_{4}\right)_{2} \mathrm{SO}_{4} 25 \%(v / v) \\
0.05 \mathrm{~mol} / \mathrm{L} \mathrm{Na}_{2} \mathrm{HPO}_{4}-\mathrm{NaH}_{2} \mathrm{PO}_{4} \\
\text { pH } 6.79\end{array}$ & $\begin{array}{l}\text { PEG4000 14\% }(w / v) \\
\mathrm{MgCl}_{2} 0.4 \mathrm{~mol} / \mathrm{L} \\
\text { Hepes } 0.05 \mathrm{~mol} / \mathrm{L} \\
\text { pH } 7.37\end{array}$ \\
\hline
\end{tabular}

a) MPD 为 2-甲基-2, 4-戊二醇

高分辨率达 $0.283 \mathrm{~nm}$. 收集条件为: 回摆角 $1^{\circ}$, 每张画面曝光时间 $6 \mathrm{~min}$, 晶体与探测器的距离 $280 \mathrm{~mm}$, 功率 $2000 \mathrm{~W}$, 共收集 170 张画面. 使用 Denzo 程序 ${ }^{[23]}$ 对数据进行处理得晶胞参数为 $a=10.80 \mathrm{~nm}, b=11.7 \mathrm{~nm}, c=18.4 \mathrm{~nm}, \alpha=\gamma=90.0^{\circ}, \beta=90.2^{\circ}$, 晶体空间群属于 $\mathrm{P} 2{ }_{1}$, 其晶胞体积 为 $2351.7375 \mathrm{~nm}^{3}$. 当含水量为 $43 \%$ 时, 每个晶胞中含 24 个 $(\alpha \beta)$ 单体. 与 Mathews 方法估算 的结果十分相似, 晶胞中一个不对称单位含有 12 个分子量为 $40.5 \mathrm{ku}$ 的 $(\alpha \beta)$ 单体. 晶型 I 晶体 的衍射数据统计见表 2 .

表 2 晶型 I 和 II 的衍射数据统计

\begin{tabular}{lcc}
\hline & 晶型 I & 晶型 II \\
\hline 总衍射点数 & 156183 & 72329 \\
独立区衍射点数 & 59891 & 13448 \\
分辨率/nm & 0.35 & 0.292 \\
完整度 $(2$ o cut off $) / \%$ & 66.7 & 74.0 \\
平均的 $I / \sigma$ & 7.3 & 8.2 \\
$R$ merge $^{\text {a) }}$ & 0.07 & 0.08 \\
\hline
\end{tabular}

a) $R$ merge $=\Sigma_{h} \Sigma_{i}\left|I(h)_{i}-\langle I(h)\rangle\right| / \Sigma_{h} \Sigma_{i} I(h)_{i}$

1.4.2 晶型 II 在恒温 $17.5^{\circ} \mathrm{C}$ 避光环境下采用气相悬滴扩散法, 按表 1 所列的晶体生长条 件, 经 1 2 周的时间获得了最大线度为 $0.4 \mathrm{~mm} \times 0.4 \mathrm{~mm} \times 0.3 \mathrm{~mm}$ 的单晶(图 4). 晶体衍射数据 采用 Mar Research Image Plate System 收集. 收集条件: 晶体与面探测器的距离为 $280 \mathrm{~mm}$, 每 个画面曝光时间 $8 \mathrm{~min}$, 回摆角 $1^{\circ}$, 功率 $2000 \mathrm{~W}(40 \mathrm{kV}, 50 \mathrm{~mA})$, 共收集了 220 张画面. 收 集到的数据使用 Denzo 程序处理得晶胞参数 $a=b=15.50 \mathrm{~nm}, c=4.03 \mathrm{~nm}$, 自动指标化得到 的结果表明该晶体属于六方晶系. 数据显示在 001 方向有明显的 $1 \neq 3 n$ 的系统消光, 在 $\mathrm{h} 00$ 和 $0 \mathrm{k} 0$ 方向无二次轴对称. 按 Laue 对称群 $6 / \mathrm{m}$ 处理衍射数据, 结果 $R$ merge 为 $8 \%$, 说明晶体空 间群属于 $\mathrm{P}_{3}$. 按 Mathews 方法估算晶胞中一个不对称单位含有 1 个分子量为 $40.5 \mathrm{ku}$ 的 $(\alpha \beta)$ 单体, 此时每单位分子量蛋白质所占体积为 $0.00345 \mathrm{~nm}^{3}$, 溶剂含量约为 $60 \%$. 晶型 II 的晶体 衍射能力最高可达到 $0.283 \mathrm{~nm}$, 但数据统计指出 $0.292 \mathrm{~nm}$ 分辨率以上的数据质量呈明显下 降趋势. 表 2 给出了藻蓝蛋白晶型 II 的数据统计结果. 


\section{2 讨论}

植物对硒酸盐的吸收有主动吸收和被 动吸收两种方式. 藻类在外界环境高浓度 的亚硒酸盐存在下，吸收、同化亚硒酸盐， 部分以无机硒形式咜存, 部分形成有机硒 化合物 ${ }^{[24 ~ 26]}$. 本试验在收获藻体后通过反 复透析, 直到检测透析液中无硒时认为透 析完全. 然后从透析过的藻中提取藻蓝蛋 白, 并充分透析(直到检测透析液中无硒)以 去掉蛋白液中的无机硒. 最后测定藻蓝蛋 白中的硒含量为 $86.8 \mu \mathrm{g} / \mathrm{g}$ 蛋白, 说明硒存 在于藻蓝蛋白中. 尽管藻蓝蛋白中硒的含 量低(相当于 22 个 $(\alpha \beta)$ 单体中含 1 个硒原 子), 这种低硒将会给确定硒原子的位置带 来很大的困难，但是在特定条件下有可能 获得一定的结构信息。即藻蓝蛋白是以 $(\alpha \beta)_{6}$ 为功能单位, 如果一个 $(\alpha \beta)_{6}$ 包含一个

图 4 钝顶螺旋藻藻蓝蛋白晶型 II 的晶体外形照片

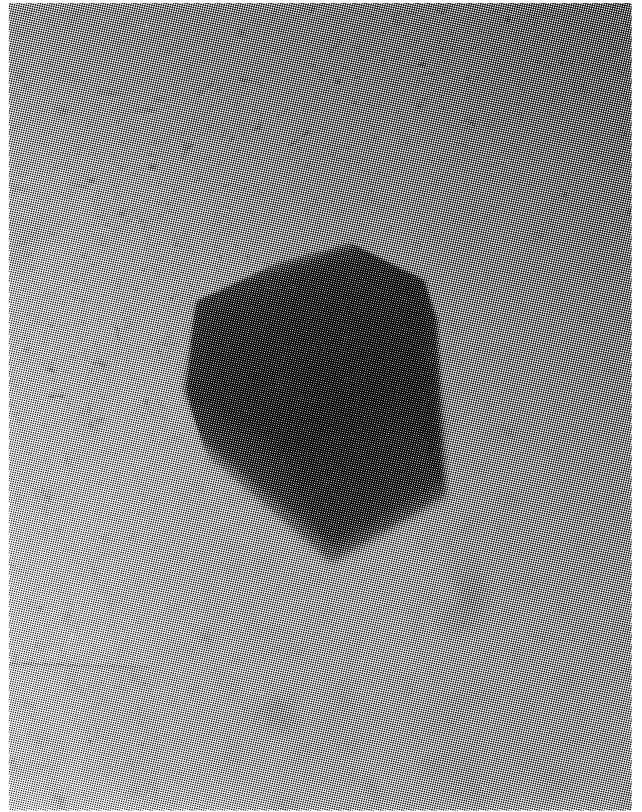
硒原子, 其占有率为 $3 \%$ 左右, 即其质量相当于一个钙原子, 其密度应有明显的表现. 然而, 还 不清楚此硒是被藻蓝蛋白吸附还是络合, 还是替代或者部分替代蛋白质中的硫, 我们希望通 过结构解析工作有助于回答这一问题，并从分子水平阐明植物硒的营养代谢机理.

对于生物样品中的硒, 目前尚无标准分析方法颁布, DAN 菼光法是国内外常用的分析方法. 为使所采用的测试方法准确可靠, 在测定硒时, 测得小麦粉硒的回收率为 $98.6 \%$. 同时还测定了 一个甘蓝成分硒标准物质(GBW08504), 其参考标准为 $0.083 \mu \mathrm{gSe} / \mathrm{g}$, 测定结果为 $0.080 \mu \mathrm{gSe} / \mathrm{g}$, 说 明测定方法是准确可靠的. 该方法的灵敏度为 $1 \mathrm{ng}$, 可以满足生物样品中微量硒的测定.

晶型 I 的晶体生长条件以 $\left(\mathrm{NH}_{4}\right)_{2} \mathrm{SO}_{4}$ 作沉淀剂, $\mathrm{Na}_{2} \mathrm{HPO}_{4}-\mathrm{NaH}_{2} \mathrm{PO}_{4}$ 为缓冲体系, 虽然作了 许多努力改变缓冲体系 $\mathrm{pH}$ 值、沉淀剂浓度、蛋白浓度, 但 $\mathrm{X}$ 射线衍射表明晶体的镶嵌现象 仍不能完全解决. 当加入 $2.5 \%$ 的 MPD 后, 晶体质量有所改善, 并获得了适合于 X 射线衍射 用的单晶. 对衍射数据初步处理, Denzo 程序中 Index 的结果表明, 晶体似乎属于正交晶系, $a=$ $10.80 \mathrm{~nm}, b=11.70 \mathrm{~nm}, c=18.40 \mathrm{~nm}, \alpha=\beta=\gamma=90^{\circ}$, 然而, 按正交晶系处理, 2.0 0.299 nm 范 围内的平均 $R$ merge 高达 $56.7 \%$. 为了正确地确定空间群, 我们按 P1 空间群处理衍射数据, 并对全球(衍射球)数据按 3 个对称面 $[\mathrm{m}(x 0 z)]$, [m( $(0 y z)]$, [m( $x y 0)]$ 找等效点并以衍射强度求 $R$ merge, 其中一个面 $[\mathrm{m}(x 0 z)]$ 的 $R$ merge 为 $10 \%$, 另外两个面的 $R$ merge 都高达 $31 \%$, 这两个面 如此高的 $R$ merge, 说明晶型 I 晶体不可能是正交晶系. 数据处理时还发现在 $0 \mathrm{k} 0$ 方向有准确可 靠的 $k \neq 2 n$ 的系统消光, 而 h00 及 001 均无系统消光, 根据 $2 n+1$ 的系统消光规律说明晶体空 间群应属于 $\mathrm{P} 2{ }_{1}$. 经过对衍射数据修正, 得 $\beta$ 角等于 $90.2^{\circ}$. 最近我们用另一颗晶体又收集了一 套数据, 按 P1 空间群处理衍射数据, 并对全球 (衍射球) 数据按 3 个对称 面 $[\mathrm{m}(x 0 z)],[\mathrm{m}(0 y z)],[\mathrm{m}(x y 0)]$ 分别找等效点并以衍射强度求 $R$ merge, 其中一个面 $[\mathrm{m}(x 0 z)]$ 的 $R$ 
merge 为 $9 \%$, 另外两个面的 $R$ merge 都高达 $37 \%$; 同样, 按 P1 空间群处理衍射数据, 并对全 球(衍射球)数据按 3 个二次轴 [h00], [0k0], [001] 分别找等效点并以衍射强度求 $R$ merge, 其结 果为一个二次轴 [0k0]的 $R$ merge 为 $9 \%$, 另外两个二次轴的 $R$ merge 都为 37\%. 由此说明晶型 $I$ 晶体不可能是正交晶系,而应该是单斜晶系, 从系统消光可以肯定其空间群为 $\mathrm{P} 22_{1}$. 空间群 为 $\mathrm{P} 2{ }_{1}$ 的藻蓝蛋白晶体在藻类藻胆蛋白中还未见有文献报道, 是一种新的晶型. 据已测定的藻 类藻胆蛋白的结构看, 藻胆蛋白多以 3 个 $(\alpha \beta)$ 单体沿三次轴组装成 $(\alpha \beta)_{3}$ 三聚体, 两个三聚体 面对面组装成一个 $(\alpha \beta)_{6}$ 六聚体. 另有报道指出 ${ }^{[27]}$, 的紧密缔合. 因此, 对于 $\mathrm{P} 2_{1}$ 空间群的藻 蓝蛋白, 每个不对称单位可能有两个 $(\alpha \beta)_{6}$ 六聚体或者 $\mathrm{P}_{2} 2_{1} 2_{1}$ 空间群的别藻蓝蛋白-连接蛋白 复合物, 两个 $(\alpha \beta)_{3}$ 三聚体存在一个接近 $90^{\circ}$ 的“边靠边” 4 个 $(\alpha \beta)_{3}$ 三聚体, 其 $(\alpha \beta)_{6}$ 六聚体或者 $(\alpha \beta)_{3}$ 三聚体之间如何组装, 对于揭示藻蓝蛋白 $(\alpha \beta)_{3}$ 三聚体、 $(\alpha \beta)_{6}$ 六聚体以及不同藻胆蛋白 之间的光能传递具有重要的理论意义, 这有待于晶体结构的测定. 目前我们正在改善晶体生 长条件, 进一步提高蛋白纯度, 有望获得更好质量的 $\mathrm{P} 22_{1}$ 晶体进行结构解析.

晶型 II 的外形为六方柱, 经 $\mathrm{X}$ 射线衍射表明晶体衍射数据质量很好, 此晶型的晶胞参数 与层理边枝藻 (Mastigocladus laminosus) 的藻蓝蛋白晶体基本一致 ${ }^{[19]}$, 空间群也属于 $\mathrm{P}_{3}$, 单位 晶胞含一个 $(\alpha \beta)_{6}$.

\section{参考文献}

1 Schwarz K, Foitz C M. Selenium as an integral part of factor 3 against dietary necrotic liver degeneration. J Am Chem Soc, 1957, 79: 3 292 3293

2 Rotruck J T, Pope A L, Ganther H E, et al. Selenium: biochemical role as a component of glutathione peroxidase. Science, 1973, 179: 588 590

3 Ehrenreich A, Forchhammer K, Tormay P, et al. Selenoprotein synthesis in E.coli. Purification and characterisation of the enzyme catalysing selenium activation. Eur J Biochem, 1992, 206: 767 773

4 Choi I S, Diamond A M, Crain P F, et al. Reconstitution of the biosynthetic pathway of selenocysteine tRNAs in Xenopus oocytes. Biochemistry, 1994, 3: 601 605

尚庆茂, 李平兰. 硒在高等植物中的生理作用. 植物生理学通讯, 1998, (4)：284 288

6 Arvy M P. Selenate and selenite uptake and translocation in bean plants. J Exp Bot, 1993, 44: $1080 \sim 1087$

7 孙景信, 王玉琦, 徐 云, 等. 小麦 POD 同工酶谱蛋白条带中硒的中子活化分析. 科学通报, 1997, (16): 171 173

8 刘曼西, 于秀芝, 孙宪洁, 等. 硒酵母成分的研究. 华中工学院学报, 1985, 13(3): 115 119

9 Bottine N R. Se containing amino acids and proteins in marine algae. Phytochem, 1984, 23: 2 445 2 452

10 王大志, 高亚辉, 程兆第. 硒对两种盐藻生长的影响及其在细胞中的累积和分布. 海洋学报, 1997, 19(5): 100 115

11 Moreno A, Bermejo R, Talavera E, et al. Purification, crystallization and preliminary X-ray diffraction studies of Cphycocyanin and allophycocyanin from Spirulina platensis. Acta Cryst, 1997, D53: 321 326

12 Brejc K, Ficner R, Huber R, et al. Isolation, crystallization, crystal structure analysis and refinement of allophycocyanin from

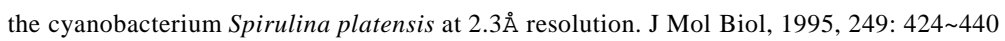

13 Ficner R, Lobeck K, Schmidt G, et al. Isolation, crystallization, crystal structure analysis and refinement of B-phycoerythrin

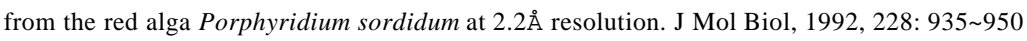

14 Jiang T, Zhang J P, Liang D C. Structure and function of chromophores in R-phycoerythrin at $1.9 \AA$ resolution. Proteins: Structure, Function, and Genetics, 1999, 34: 224 231

Chang W R, Tao J, Wan Z L, et al. Crystal structure of R-phycoerythrin from Polysiphonia urceolata at $2.8 \AA$ resolution. J Mol Biol, 1996, 262: 721 731 
16 Ficner R, Huber R. Refined crystal structure of phycoerythrin from Porphyridium cruentum at $0.23 \mathrm{~nm}$ resolution and localization of the $\gamma$ subunit. Eur J Biochem, 1993, 218: 103 106

17 Fisher R G, Woods N E, Fuchs H E, et al. Three-dimensional structures of C-phycocyanin and B-phycoerythrin at $5 \AA$ resolution. J Biol Chem, 1980, 255: 5 082 5 089

18 Duerring M, Huber R, Bode W. Refined three-dimensional structure of phycoerythrocyanin from the cyanobacterium

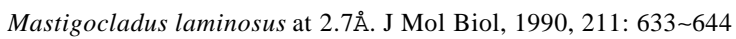

19 Schirmer T, Bode W, Huber R. Refine three-dimensional structures of two cyanobacterial C-phycocyanins at 2.1 and $2.5 \AA$ resolution. A common principle of phycobilin-protein interaction. J Mol Biol, 1987, 196: 677 695

20 Duerring M, Schmidt G B, Huber R. Isolation, crystallization, crystal structure analysis and refinement of constitutive Cphycocyanin from the chromatically adapting cyanobacterium Fremyella diplosiphon at $1.66 \AA$ resolution. J Mol Biol, 1991, 217: 577 592

21 Liu J Y, Jiang T, Zhang J P, et al. Crystal structure of allophycocyanin from red algae Porphyra yezoensis at $2.2 \AA$ resolution. J Biol Chem, 1999, 274(24): 16 945 16952

22 Watkinson J H. Fluorometric determination of selenium in biological material with 2,3-diaminonaphthalene. Analytical Chemistry, 1966, 38: 92 94

23 Otwinowski Z, Minor W. Processing of X-ray diffraction data collected in oscillation mode. Methods in Enzymology, 1997, 276: $307 \sim 326$

24 Asher C J, Butler G J, Peterson P J. Selenium transportion room system of tomato. J Exp Bot, 1977, 28: 279 291

25 Wong D. Effects of selenite and selenate on the growth and motility of seven species of marine microalgae. Can J Fish Aquat Sci, 1991, 48: 1 193 1 200

26 Zhang G H. Se uptake and accumulation in marine phytoplankton and transfer of Se to the clam puditapes philippnarum. Marine Environ Res, 1990, 30: 179 190

27 Reuter W, Wiegand G, Huber R, et al. Structural analysis at $2.2 \AA$ of orthorhombic crystals presents the asymmetry of the allophycocyanin-linker complex, AP. $\mathrm{L}_{\mathrm{c}}^{7,8}$, from phycobilisomes of Mastigocladus laminosus. Proc Natl Acad Sci USA, 1999, 96: $1363 \sim 1368$ 